
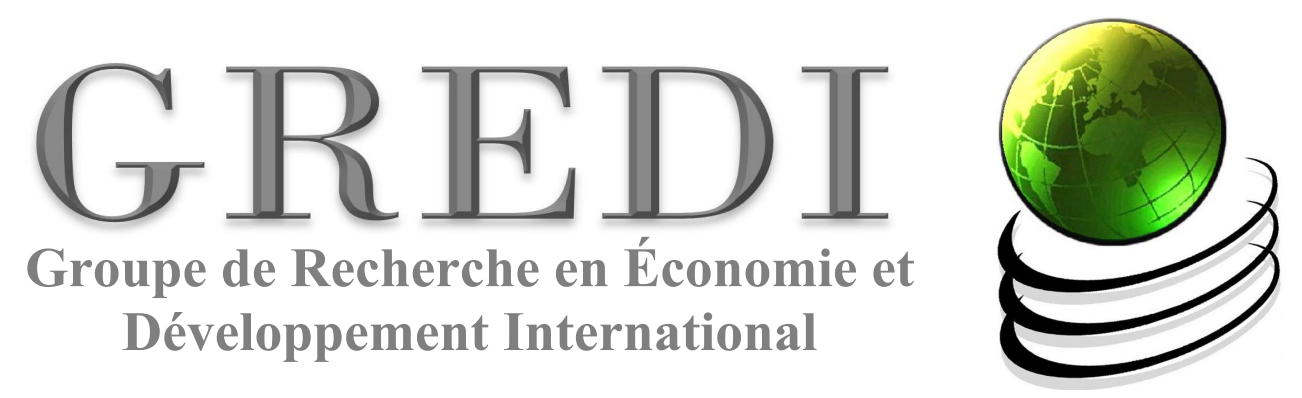

\author{
Cahier de Recherche / Working Paper \\ 12-01
}

Price and quality decisions under network effects

Noemí Navarro 


\title{
Price and quality decisions under network effects*
}

\author{
Noemí Navarro ${ }^{\dagger}$ \\ Université de Sherbrooke
}

February 2012

\begin{abstract}
I analyze monopoly pricing and quality decisions under network effects. High quality premium and low quality punishment are found to depend on how the impact of marginal costs on quality relates to the intensity of the network effect and the optimism of the producer about final demand. More precisely, marginal costs have to be low enough (but not too low) with respect to the intensity of the network effects and/or the optimism about final demand so that higher prices reflect higher quality. A similar conclusion can be drawn about incentives for quality provision, whenever quality is considered endogenous together with price.
\end{abstract}

Keywords: Network effects, optimal pricing, quality provision

JEL Classification: L12, L14, L15, D42, D82, D83, C7.

\section{Introduction}

This paper analyzes pricing and quality decisions of a monopolist when there are network effects on the consumers' side. By network effects on the consumers' side I mean that consumers affect each other's willingness-to-pay for the good, either in a positive or a negative way. To the three classical examples of network effects by Katz and Shapiro (1985), namely (i) direct physical effects of the number of purchasers on the value of the product (telephone, email, file sharing

${ }^{*}$ The model here is a generalization of previous working papers, "Asymmetric information, word-of-mouth and social networks: From the market for lemons to efficiency", CORE discussion paper 02/06, written during my stay at CORE as a research fellow, and "Quality provision under referral consumption", written during my stay at Málaga University. I gratefully acknowledge Alessandro Citanna and an anonymous referee whose comments completely re-shaped (and re-focused) the paper. Financial support from the Chaire CN en Économie et Intermodalité des Transports, CRT, Université de Montréal, from research projects SEJ-556 and SEJ-1645 from Junta de Andalucía and from research projects ECO 2008-03674/ECON and ECO 2011-29355 from the Spanish Ministry of Science and Innovation are gratefully acknowledged. Part of this work was developed while visiting HEC Lausanne and was completed while visiting CIRPÉE, UQàM. I am grateful for their hospitality. The usual disclaimer applies.

†Université de Sherbrooke, Département d'Économique and GREDI, email: noemi.navarro@usherbrooke.ca 
programs), (ii) the hardware/software paradigm (associated to strong complementarities) and (iii) quality and availability of a post-purchase service (again, strong complementarities), a fourth one has more recently been added: a market for an experience good where consumers use word-of-mouth to learn about the quality of such a good. While in the first three examples network effects are positive, in this work I consider positive network effects as well as negative ones, as in the fourth example above. Word-of-mouth will decrease willingness-to-pay when the actual quality of the experience good is lower than the initial expectation. Negative network effects could also appear in the market for cars: If too many people buy (and use) a car, traffic congestion will make the use of a car less valuable.

In this paper I explore the conditions under which higher (positive) network effect implies a higher price and whether lower (negative) network effect implies a lower price. Some seminal papers analyzing pricing decisions for experience goods have also explored conditions for prices to act as signals for quality, typically characterizing separating equilibria (Wolinsky 1983, Milgrom and Roberts 1986, Liebeskind and Rumelt 1989, or Bagwell and Riordan 1991). In Shapiro's work (Shapiro 1982, 1983a, 1983b) higher prices are to be interpreted not only as signals for quality but as a premium for quality provision too, though quality is not endogenously chosen. Shapiro (1983b) proved that price taking behavior with entry at any quality level (sort of a perfect competition setting) also results in a price-cost mark-up that increases in quality.

There is also an extensive literature on network effects sice the classical model of telecommunication demand by Rohlfs (1974). It notably includes strategic analysis of compatibility standards (among others, Farrell and Saloner 1985 and 1986, Katz and Shapiro 1985, 1986, 1992 and 1994 and Bessen and Farrell 1994) and, closer to what I do here, dynamic monopoly pricing under network effects (Shapiro 1983a, Bensaid an Lesne 1996, Vettas 1997 or Cabral, Salant and Woroch 1999, among others). ${ }^{1}$ In all these articles network effects on the demand side are modeled as an increasing willingness-to-pay for consumers in the size of the market, as if the way buyers interact via the social network structure is a black box. In this work, I model those interactions via a explicit social network without eliminating the possibility of indirect network effects, where the willingness-to-pay increases in the number of buyers. These network effects are heterogeneous in the sense that one market size could affect two consumers differently. Individual willingness-to-pay is affected by who the other buyers are and also by how these buyers are related in the given social network, therefore capturing direct network effects. As usual, network effects shape the possible final (or total) demand that the producer may expect to arise for each combination of quality and price. The model presented here shares in common, particularly with the work by Katz and Shapiro (1985), the notion of a fulfilled or rational expectations equilibria on the consumers side.

I find that network effects have to be big enough with respect to the impact of quality on marginal costs so that a high quality producer extracts a premium from the positive network

\footnotetext{
${ }^{1}$ See Shy (2011) for a recent revision in network economics from an industrial organization perspective. A very well known and influential review of network effects is Economides (1996).
} 
effect. Nevertheless, if the impact of quality on marginal costs is too low, the monopolist prefers charging a low price and capture all the market than charging a high price to capture smaller expected demand, typically the agents with higher willingness-to-pay (influenced by the network effect). If the network effect is negative (congestion), the impact of quality on marginal costs also has to be low enough so that monopoly price is low in order to increase expected demand. Most surprisingly, the producer of a congested good will always stay in the market while the producer of a good with a positive network externality exits the market if the impact of quality on marginal costs is high enough.

If the monopolist is allowed to choose quality previous to the pricing decision, ${ }^{2}$ he will choose the highest possible quality if (i) the impact of higher quality on marginal costs is low enough, compared to the structure of network effects and the optimism of the producer about final demand, and (ii) the highest possible quality is high enough. The latter is due to the fact that the price-cost mark-up, when price extracts a premium for quality, is increasing in the level of quality. If the impact on marginal costs is intermediate, the quality level that is chosen will imply a zero network effect. Finally, if the impact of higher quality on marginal costs is high enough, the monopolist chooses to produce a good with the worse (and negative) network effect.

Liebeskind and Rumelt (1989) and Bagwell and Riordan (1991) consider quality decision as endogenous in the context of dynamic pricing of an experience good. Two features are required to sustain high quality levels in equilibrium: (i) repeat purchase with learning from own experience, and (ii) boycotting in case of low quality. In Shapiro (1983b), reputation and the mark-up price associated to it are the two features sustaining high quality levels in equilibrium. My analysis considers markets where only the mark-up price is available, and the question in order is whether the network effect, with the resulting effect on prices, is sufficient to provide incentives for (costly) higher quality to the producer. This happens only when the impact of quality on marginal costs are low enough.

Two articles by Nikolaos Vettas (1997 and 1998) and one by Carl Shapiro (1983a) are probably, among the literature for experience goods, the closest to the one here. Vettas (1997) and Shapiro (1983a) analyze dynamic monopoly pricing. In both settings consumers learn the value of the good from past sales. In particular, Shapiro (1983a) assumes that consumers immediately update their expectations about quality once the good has been purchased and Vettas (1997) assumes that the probability for a consumer to learn the real quality is an increasing function of past sales. Shapiro (1983a) shows that the high-quality monopolist chooses a higher price than the low-quality monopolist and Vettas (1997) shows that the high-quality monopolist obtains a higher expected profit than the low-quality monopolist. Vettas (1998) analyzes entry decision

\footnotetext{
${ }^{2}$ Choosing higher quality in this context means choosing higher willingness-to-pay for consumers keeping the structure of network effects as given. We can think for example of the automobile industry. Positive network effects are related to the availability of technical and post-purchase services (indirect network effects through complementarities) while negative network effects have to do with road congestion and air pollution. Making a car that is less pollutant is more costly (in terms of marginal costs per unit of a less polluting car) than making a car that is a complete environmental disaster.
} 
under price-taking behavior on both sides of the market. Consumers who have not acquired the good can observe the behavior of all the other consumers. Like that, a lower bound for quality (given by price) can be inferred when a repeated purchase is observed. Market clearing prices are increasing in time when quality is higher than initial expectation, while they are decreasing when quality is lower than initial expectation. Shapiro (1983a) and Vettas (1997) lie probably closer to this work than Vettas (1998), due to the fact that there is a (monopolist) price taking decision. Vettas (1998) learning process, though, looks more similar to the network effect on the consumers' side here than Shapiro's (1983a) and Vettas (1997) learning processes. Vettas (1998) serves as an example of network effects due to word-of-mouth in markets for experience goods, adding up to the three classical examples by Katz and Shapiro (1985) mentioned above, but in which the network of consumers is the complete one. In my setting, the network of consumers is allowed to be incomplete and network effects can represent any of the four examples mentioned at the beginning of this introduction.

The rest of the paper is organized as follows. Section 2 states the model, while Sections 3 and 4 present the main results in terms of price and quality (or network effect), respectively. Some examples are shown. A detailed version of all proofs are in the Web Appendix, while a condensed version of the two main propositions is offered in the Appendix here.

\section{The Model}

There is one good produced by a single agent, referred to as the producer or the monopolist. The producer chooses a price, given a certain quality. ${ }^{3}$ Then consumers choose simultaneously to consume or not, given the prices and the social structure.

\subsection{Consumers: Utility}

The good with quality $\theta$ is available for consumers at a market price denoted by $p$. Consumers are risk neutral, need at most one unit of the good, and their willingness-to-pay depends on the other consumers who buy the good and how they relate to each other through the social network structure. Thus, the utility function for each consumer $i$ can be written as

$$
U_{i}(p)= \begin{cases}W_{i}(S, g)-p, & \text { if } i \text { buys the good, } \\ 0, & \text { otherwise }\end{cases}
$$

where $W_{i}(S, g)$ is the willingness-to-pay when the consumers' social network structure is equal to $g$ and the set $S \subseteq N$ is the set of consumers who acquire the good.

\footnotetext{
${ }^{3}$ I will eventually analyze the case of endogenous quality choice.
} 


\subsection{Consumers: The Social Structure}

The consumers' social network is represented by an undirected graph $g$. For simplicity and since the set $N$ is considered to be fixed I will treat $g$ as a set of links or unordered pairs $i j$, where $i, j \in N$, and $i \neq j$. The notations $i j \in g, i j \notin g, g \cup i j$ and $g \backslash i j$ have the usual meanings. ${ }^{4}$ I denote by $g^{N}$ the complete graph, meaning the graph where all possible unordered pairs are listed, and by $g^{i}$ the graph where agent $i$ is directly connected to everybody else and everybody else is only connected to agent $i$. This structure $g^{i}$ is normally called the star with center in agent $i$. A regular network is a structure such that all consumers have the same number of connections (degree of connectivity). A circle and the complete network are examples of regular networks with degree equal to 2 and to $n-1$, respectively. A core-periphery structure will be a network in which a subset $A$ of consumers, called the core, with $\alpha=|A|$, are directly connected to each other. The rest of consumers, called the periphery, will be connected to some of the consumers in the core. Figure 1 shows different social structures for $n=5$.

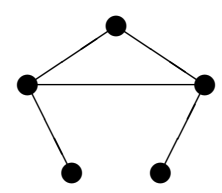

A core with 3 agents

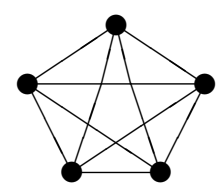

The complete graph

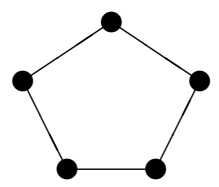

A circle

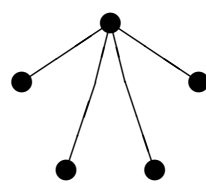

A star

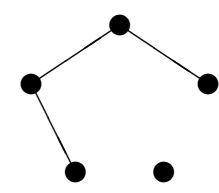

A line with 4 agents

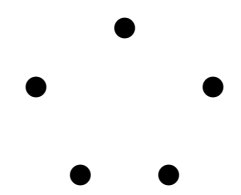

The empty graph

FIGURE 1

Given a graph $g$, a group of consumers $S \subseteq N$ is called a component of $g$ if: (1) for every two consumers in $S$, there is a path, that is, a set of consecutive links in $g$ connecting them, and (2) for any consumer $i$ in $S$ and any consumer $j$ not in $S$, there is no path in $g$ which connects them. Let $\mathcal{C}(g)$ be the set of components of $g$. Note that $\mathcal{C}(g)$ is a partition of $N$. In Figure 1 above, the line with four agents has two components, one where there is one isolated consumer and the other one with four of them together. The empty graph has five components, which are all singletons. The other graphs have only one component.

A graph $g$ is connected if $\mathcal{C}(g)=\{N\}$. Therefore, in the examples in Figure 1 above only the line with four agents and the empty graph are not connected.

\footnotetext{
${ }^{4}$ See for example Jackson and Wolinsky (1996) or Jackson (2008)
} 
Two disjoint sets of consumers $S$ and $S^{\prime}$ are said to be connected if they belong to the same component of $g$. Note that these sets can be singletons.

\subsubsection{Network effects on utility}

Let $\theta_{0}$ be the willingness-to-pay in the absence of network effects, i.e., $\theta_{0}=W_{i}(\emptyset, \emptyset)$, and let $\theta$ be the willingness-to-pay under the highest network effect, i.e., $\theta=W_{i}\left(g^{N}, N \backslash\{i\}\right)$. If $\theta>\theta_{0}$ the network effect is positive and if $\theta<\theta_{0}$ the network effect is negative. The parameter $\theta$ is thus our measure of quality of the good.

For the intermediate cases, the willingness-to-pay for a consumer $W_{i}(S, g)$ is written in terms of a family of functions $\lambda_{i}(S, g) \in[0,1]$, with $S \subseteq N$ such that

$$
W_{i}(S, g)=\lambda_{i}(S, g) \theta+\left(1-\lambda_{i}(S, g)\right) \theta_{0} .
$$

These functions $\lambda_{i}(S, g)$ have the following properties:

1. $\lambda_{i}(S, g) \leq \lambda_{i}(S \cup\{j\}, g)$, for any $j \notin S$, and $\lambda_{i}(S, g) \leq \lambda_{i}(S, g \cup j k)$, for any $j k \notin g$.

2. $\lambda_{i}(\emptyset, g)=0$ for all $i \in N$.

3. $\lambda_{i}(S, g)=\lambda_{i}(S \backslash\{i\}, g)$, for any $S \subseteq N$ and any $i \in S$.

Representing consumer's willingness-to-pay in terms of a convex combination implies that (i) if $\theta=\theta_{0}$ there is no network effect on willingness-to-pay, (ii) network effects are either always positive or always negative, and (iii) their intensity is non-decreasing after a new consumer acquires the good or the network gets more connected. Furthermore, Property 2 says that there are no possible network effects if no one buys the good and Property 3 means that the own network effects are negligible or ignored.

This setting here of network effects on the demand side contrasts with the more traditional modeling of network effects. In the traditional models of network effects consumers are uniformly located in an interval and willingness-to-pay increases in the expected number of other buyers. For example, in Rohlfs (1974) each consumer has a location $x$ in the interval $[0,1]$ and obtains a utility $(1-\beta x) \alpha q^{e}-p$, where $q^{e}$ is the expected number of total consumers, and $\beta$ and $\alpha$ are parameters that measure, respectively, the idiosyncratic network effect (depending on location) and the total general network effect. Katz and Shapiro (1985) locate consumers in $(-\infty, A]$, with each consumer $x$ obtaining a utility from buying equal to $x+v\left(q^{e}\right)$, where $v$ is an increasing, concave function of the expected number of consumers. Note that these specifications allow for consumer's heterogeneity with respect to the network effect (Rohlfs) or with respect to the individual willingness-to-pay in the absence of network effects (Katz and Shapiro), but they do not take into account the relative position in the (given) social network, probably due to the atomless nature of consumers. The specification I propose here allows for heterogeneity in 
network effects even if these depend only on the total value of buyers, as it has been the case in the literature of network effects, and also captures direct network effects where the influence on the consumer's willingness-to-pay depend on who the other buyers are and how closely related they are to the given consumer.

\subsection{Production}

For the sake of simplicity, the good with willingness-to-pay at highest network effect $\theta \geq 0$ is produced at a marginal cost of $c \theta$, for $0<c<1$, with zero fixed costs. By this I mean in particular that a product that generates positive network externalities $\left(\theta>\theta_{0}\right)$ is more costly to produce than a product that does not generate network effects $\left(\theta=\theta_{0}\right)$ or a product that generates congestion effects $\left(\theta<\theta_{0}\right)$.

The expected profit to the producer is then given by

$$
\pi(p)=q^{d}(p)[p-c \theta]
$$

where $q^{d}(p)$ is the expected number of consumers buying one unit of the good (and therefore the expected demand) when the producer chooses price $p .^{5}$

\subsection{Equilibrium concept}

The equilibrium concept is defined backwards, formalizing first the consumers' possible demands from the producer's point of view. The producer's objective will consist of maximizing expected profit.

Definition 2.1 Given $\theta, \theta_{0}, g$, and $p$, a subset of consumers $B \subseteq N$ is called a demand configuration if (i) for every $i \in B: \lambda_{i}(B, g) \theta+\left(1-\lambda_{i}(B, g)\right) \theta_{0} \geq p$, and (ii) for every $i \notin B$ : $\lambda_{i}(B, g) \theta+\left(1-\lambda_{i}(B, g)\right) \theta_{0}<p$.

Let $Q(p)$ denote the set of all possible demand configurations $B \subseteq N$. In general there may be multiplicity of equilibria on the consumers side, which implies that $Q(p)$ typically consists of several subsets of consumers. Note that the set of equilibria $Q(p)$ depends on $p$ since the willingness-to-pay of any buyer in $B$, for each $B$ in $Q(p)$, has to be greater or equal than $p$. Consumers, though, do not build up beliefs about the purchasing decisions of the other consumers based on the price. They take the identity of the buyers in $B$, as well as price, as given. Afterwards, we require such a $B$ to be consistent with the utility maximizing behavior of each member in $B$ and of each member not in $B$. This is why one could say that a demand configuration is a rational expectations equilibria on the consumers side.

\footnotetext{
${ }^{5}$ The expected number of consumers depends on all the elements of the willingness-to-pay but to avoid abuse of notation they are omitted from the brackets.
} 
I define the equilibrium as a one shot on the consumers side since (i) what is relevant for the producer is the number of consumers who, at the end of the process, have acquired the good, and not how that happened, and (ii) I do not need to specify an ad-hoc dynamics behind the purchasing process, since there is no possibility for the producer to re-adjust the price. The demand configuration refers to the consumers decision during the time the price does not change.

Definition 2.2 The producer has network-consistent beliefs if, given $\theta, \theta_{0}$, and $c$, the expected number of consumers $q^{d}(p)$, for any choice of $p$, can be written as the convex combination

$$
\sum_{B \in Q(p)} \rho(B, Q(p))|B|
$$

where $\rho(B, Q(p))>0$, for all $B \in Q(p)$, and $\sum_{B \in Q(p)} \rho(B, Q(p))=1$.

Network consistent beliefs is the notion that allows me to deal with the problem of multiplicity of equilibria on the consumer's side. In other contexts, Arganziano (2008), Ambrus and Arganziano (2009) or Fudenberg and Tirole (2000) have solved the problem of multiplicity of equilibria by applying equilibrium refinements. Here, a refinement would select a member $B$ in $Q(p)$ with probability 1 . I have no reason to assume that a monopolist would use a particular refinement over another one when computing expected demand. His own beliefs would drive his pricing decision (and eventually quality if there is a decision of that type). All I am assuming

is that the producer believes that his final demand would be a set of consumers that form a buyers' configuration on the consumers' side.

Definition 2.3 An equilibrium is a pair $\mathcal{E}=\left(p^{*}, Q^{*}\right)$, where $p^{*}$ is a non-negative real number and $Q^{*}$ consists of subsets of consumers such that

1. The producer chooses price $p^{*}$ maximizing expected profit given network-consistent beliefs (Definition 2.2).

2. $Q^{*}=Q\left(p^{*}\right)$.

\section{Results I: Pricing with an exogenous quality}

\subsection{General $\lambda$ functions}

The following proposition characterizes the pricing decision by the producer for given $\theta, \theta_{0}$ and $c$, for simplicity denoted $p^{*}$. I characterize such a pricing decision in terms of the family of $\lambda$ functions and therefore need to introduce the following definitions. For $B \subseteq N$, let $\lambda^{\max }(B)=$ $\max _{i \in B} \lambda_{i}(B, g)$ and $\lambda^{\min }(B)=\min _{i \in B} \lambda_{i}(B, g)$ be the biggest and the smallest network effect 
for consumers inside $B$, respectively, and let $\lambda^{\max }(\neg B)=\max _{i \notin B} \lambda_{i}(B, g)$ and $\lambda^{\min }(\neg B)=$ $\min _{i \notin B} \lambda_{i}(B, g)$ be the biggest and the smallest network effect, resp., for consumers outside $B$. By definition, $\lambda^{\max }(\neg \emptyset)=\lambda^{\min }(\neg \emptyset)=0$ and $\lambda^{\min }(B)=\lambda^{\max }(B)=0$ if $B$ is a singleton (recall that by Property 3 agents ignore own network effects). Note that $\lambda^{\max }(N) \theta+\left[1-\lambda^{\max }(N)\right] \theta_{0}$ is the highest willingness-to-pay by consumers given the network $g$. This convex combination is in general different from $\theta$, unless $g$ is such that $\lambda_{i}(N, g)=1$ for at least one $i$.

Proposition 3.1 Assume the producer has already chosen a quality level $\theta$. We have to distinguish three cases.

1. If $\theta=\theta_{0}$ then the price chosen by the monopolist is equal to $\theta_{0}$ and all consumers buy the product.

2. If $\theta>\theta_{0}$ there are two threshold levels for cost $c_{1}<\frac{\theta_{0}}{\theta}<c_{2} \leq 1$ such that:

(a) If $c \leq c_{1}$ the monopolist chooses a price $p^{*}=\theta_{0}$

(b) If $c_{1} \leq c \leq c_{2}$ the monopolist chooses a price $p^{*}>\theta_{0}$ with $p^{*}=\lambda^{\min }(B) \theta+$ $\left(1-\lambda^{\min }(B)\right) \theta_{0}$ for some $B \subset N$ in $Q\left(p^{*}\right)$ with $\lambda^{\max }(\neg B)<\lambda^{\min }(B)$.

(c) Finally, if $c \geq c_{2}$ then the monopolist prefers not to produce.

3. If $\theta<\theta_{0}$ there is a threshold level for cost $c_{3}>0$ such that

(a) If $c \leq c_{3}$ the monopolist chooses a price $p^{*}<\theta_{0}$ with $p(\theta)=\lambda^{\max }(B) \theta+\left(1-\lambda^{\max }(B)\right) \theta_{0}$ for some $B \subset N$ in $Q\left(p^{*}\right)$ with $\lambda^{\max }(B)<\lambda^{\min }(\neg B)$, and

(b) If $c_{3} \leq c$ the monopolist chooses $p^{*}=\theta_{0}$.

In this case, the monopolist always produces.

Surprisingly, the "premium to quality" for positive network externalities results when the impact of quality on marginal costs lies in a middle range. If such an impact is too high, the monopolist prefers not to produce at all. If it is too low, the monopolist prefers fixing a price equal to $\theta_{0}$ and be sure to sell to all consumers. Recall that for the case of positive externalities a price higher than $\theta_{0}$ could result in no one buying the product. There is a trade-off between higher price and lower demand that depends on the network effect on demand and the particular structure of the producer beliefs. If expectations about no one buying the product are low enough at the optimum (i.e., the monopolist is optimistic enough) increasing the price is always worth it. Technically, all this means that the threshold $c_{1}$ could be negative for certain values of the parameters (see next corollary). The corresponding equilibrium on the consumers side for the optimal choice has to satisfy that the network effects on buyers are bigger than the network effects on the non-buyers. Otherwise, either a non-buyer is willing to pay the price or a buyer paid too much, which cannot happen in equilibrium. 
Similarly, the punishment for "congestion effects" results for a low range of the impact of quality on marginal costs. Again, charging lower prices implies a higher expected demand. This effect depends, as before, on the network effects on the demand side and on the structure of producer beliefs. The smaller the negative effect on utilities, the bigger the incentives to lower the price for the producer, as more consumers require a smaller reduction of the price. As in the case of positive externalities, the more optimistic the producer's beliefs about demand size, the bigger his incentives to lower the price. Technically, all this means that $c_{2}$ could be bigger than 1 . Finally, the corresponding equilibrium on the consumers side for the optimal choice has to satisfy that the network effects on buyers are smaller than the network effects on any of the non-buyers (as it is negative). The price has to be high enough so that non buyers do not want to buy, but low enough so that buyers agree to purchase the product.

Corollary 3.2 Consider first the case where $\theta>\theta_{0}$. If the producer is optimistic enough about demand size at the optimum or if the network effect on willingness-to-pay is big enough, we have that $p^{*}>\theta_{0}$. Respectively, when $\theta<\theta_{0}$ we obtain an equilibrium price $p^{*}<\theta_{0}$, provided either the producer is optimistic enough about demand size at the optimum (as before) or if the network effect on willingness-to-pay is small enough.

\subsection{Particular cases}

For any given set of buyers $B$ let the direct neighbors of a given consumer $i$ who belong to $B$ be denoted by $B_{i}$. Formally, $B_{i}=\{j \in B$ such that $i j \in g\}$.

\subsubsection{The Telephone}

Let us assume a good such that

1. Each consumer will typically care of how many other directly connected consumers are also using the good.

2. The utility extracted from the good is equal to zero if no direct friend is owning a good.

These two ideas mean that

1. $\lambda_{i}(B)=\lambda_{i}\left(B^{\prime}\right)$ if $B_{i}$ and $B_{i}^{\prime}$ are the same.

2. $\theta_{0}=0$

We can further simplify our family of lambda functions assuming symmetry, anonimity or homogeneity of network effects as:

3. $\lambda_{i}(B)=f\left(\left|B_{i}\right|\right)$, where $f($.$) is an increasing function.$ 
The condition for $B \in N$ being a demand configuration is $\lambda^{\max }(\neg B)<\lambda^{\min }(B)$, which in this case means $\max _{i \notin B}\left|B_{i}\right|<\min _{i \in B}\left|B_{i}\right|$. Let us analyze different network structures.

\section{The star network}

In the case of the star network, any $B$ including the center of the star and not including part of the non-center consumers will satisfy that (i) $\max _{i \notin B}\left|B_{i}\right|=1$, provided $B \neq N$, and (ii) $\min _{i \in B}\left|B_{i}\right|=1$, provided $B \neq \emptyset$. Therefore, the monopolist has two possibilities if $\theta>\theta_{0}=0$. He could charge the maximal price among the ones that result in $Q(p)=\{N, \emptyset\}$, which is $p^{*}=f(1) \theta$, or he does not produce at all. Recall that charging a price equal to 0 implies that all the $n$ consumers buy the product for sure (as $\theta_{0}=0$ ) but yields negative profits. In the first case, he obtains a profit equal to $\rho n(f(1)-c) \theta$, where $\rho$ is the probability that the producer believes that $N$ will be the consumers equilibrium out of $\{N, \emptyset\}$. The optimal decision therefore is $p^{*}=f(1) \theta>0$ if $\rho>0, \theta>0$ and $f(1)>c$.

\section{A regular network of degree $k$}

This category includes the complete network (when $k=n-1$ ) and the circle $(k=2)$. In the case of the regular network, an equilibrium configuration cannot have "holes" according to the network. The condition $\max _{i \notin B}\left|B_{i}\right|<\min _{i \in B}\left|B_{i}\right|=1$ means in words that, in equilibrium, nonbuyers, if they were to buy the good, are worse than buyers. Given the regularity of the network, the highest willingness-to-pay of a non-buyer is at least as good as the worse willingness-to-pay of the buyers, as far as the set of buyers $B$ is nonempty and different from $N$.

For example, let us take the complete network. The number $\max _{i \notin B}\left|B_{i}\right|$ corresponds to $\left|B_{i}\right|$ since everybody is directly connected to everybody else. On the other hand, $\min _{i \in B}\left|B_{i}\right|$ is equal to $\left|B_{i}\right|-1$, because each member of $B$ is connected to all of the other members of $B$.

In the case of the circle, the number $\max _{i \notin B}\left|B_{i}\right|$ corresponds to a direct neighbor of a buyer, which always exists, given the regularity of the circle, and as far as there is at least one consumer not in $B$. Therefore, $\max _{i \notin B}\left|B_{i}\right|=1$. But the number $\min _{i \in B}\left|B_{i}\right|$ can only be equal to 2 (i.e., bigger than 1) when $B=N$. Otherwise $\min _{i \in B}\left|B_{i}\right|=1$.

For the sake of brevity, let us assume $n$ and $k$ are even. ${ }^{6}$ Buyers must be adjacent to each other in equilibrium (since otherwise non-buyers surrounded by buyers have a willingness-to-pay as good as the buyers). The number $\max _{i \notin B}\left|B_{i}\right|$ corresponds to the non-buyer adjacent to a buyer in one of the extremes of $B$, who is at least connected to $\frac{k}{2}$ people (say on his right). If $B$ is big enough, he could be connected to some of the buyers who are his direct connections on the other side (say on his left). If $|B| \leq \frac{k}{2}$, then $\max _{i \notin B}\left|B_{i}\right|=|B|$ and $\min _{i \in B}\left(B_{i}\right)=|B|-1$. If $\frac{k}{2}<|B|<n-\frac{k}{2}$, then $\max _{i \notin B}\left|B_{i}\right|=\frac{k}{2}$ and $\min _{i \in B}\left|B_{i}\right|=\frac{k}{2}$ since the consumer at one of the extremes of $B$ can only be connected to buyers on one of his two sides (say his right too). Finally, if $n-\frac{k}{2} \leq|B|$, then $\max _{i \notin B}\left|B_{i}\right|=k-(n-|B|-1)$, i.e., all direct connections minus

\footnotetext{
${ }^{6}$ The argument is similar otherwise, assuming the degree $k$ is compatible with a population of size $n$, and replacing $\frac{k}{2}$ by $\frac{k-1}{2}$
} 
all the other non-buyers, and $\min _{i \in B}\left(B_{i}\right)=k-(n-|B|)$, i.e, all direct connections minus all the non-buyers.

Again, the monopolist has two possibilities if $\theta>\theta_{0}$ : charging the maximal price that obtains $Q(p)=\{N, \emptyset\}$, which is $p^{*}=f(k) \theta$, or not producing at all. The optimal decision therefore is $p^{*}=f(k) \theta>0$ if $\rho>0, \theta>0$ and $f(k)>c$.

\section{A core-periphery structure}

Recall that the size of the core is equal to $\alpha$. It is easy to see that, apart from $N$ or the empty set as before, we can also obtain equilibria in which only the core is buying the telephone for positive prices. Therefore, the monopolist can choose to charge a price equal to $f(n-1) \theta$, equal to $f(\alpha-1) \theta$ or not to produce at all. The corresponding sets of configuration equilibria are $Q(f(n-1) \theta)=\{N, \emptyset\}$ and $Q(f(\alpha-1) \theta)=\left\{A \cup P_{1}, \emptyset\right\}$, where $P_{1}$ is the (possibly empty) set of periphery consumers connected to $\alpha-1$ members of the core $A$. Assume that $\rho_{n}=\rho(N,\{N, \emptyset\})$ and $q_{\alpha}^{e}=\rho\left(A \cup P_{1},\left\{A \cup P_{1}, \emptyset\right\}\right)\left|A \cup P_{1}\right|$. Hence, $p^{*}=f(\alpha-1) \theta$ if $c<f(\alpha-1)$ and

$$
f(\alpha-1)-c \geq \frac{\rho^{n} n}{q_{\alpha}^{e}}(f(n-1)-c) .
$$

Note that this can only be true if $q_{\alpha}^{e}>\rho_{n} n$. Otherwise, $p^{*}=f(n-1) \theta$ if $c<f(n-1)$.

\subsubsection{A standardized market (indirect effects)}

Let us now assume a good such that

1. Each consumer cares of how many other consumers in total are also using the good.

2. The utility extracted from the good is equal to zero if nobody else is owning the good.

These two ideas imply that

1. $\lambda_{i}(B)=\lambda_{i}\left(B^{\prime}\right)$ if the cardinality of $B_{i} \backslash\{i\}$ is equal to the cardinality of $B_{i}^{\prime} \backslash\{i\}$.

2. $\theta_{0}=0$

As before, let us assume some anonimity in the lambda functions so that we can simplify our family of lambda functions as

3. $\quad \lambda_{i}(B)=f(|B|)$, if $i \notin B$, and $\lambda_{i}(B)=f(|B|-1)$, if $i \in B$, where $f($.$) is an increasing$ function.

Given our assumptions for a standardized market, $\lambda^{\max }(\neg B)<\lambda^{\min }(B)$ implies that, for any $B$ that is non empty and different from $N, \max _{i \notin B}|B|<\min _{i \in B}|B|-1$, which is never true. Hence, in equilibrium we can either have everybody buying the good or nobody buying the good, and, independently of the network structure, the optimal decision is then $p^{*}=f(n-1) \theta>0$ whenever $\rho=\rho(N,\{N, \emptyset\})>0$ and $c<f(n-1)$. 


\subsubsection{File sharing}

File sharing is a mix of the telephone case and the standardized market case.

1. Each consumer typically cares of how many other consumers in total are also using the good.

2. Each consumer also cares of how many of his friends or direct connections are also using the same good.

3. The utility extracted from the good is equal to zero if nobody else is using the good.

These ideas can be captured by a combination of the telephone and the standardized market. If we simplify the problem by imposing homogeneity we obtain

$$
\lambda_{i}(B)=\gamma f^{T}\left(\left|B_{i}\right|\right)+(1-\gamma) f^{S}(|B \backslash\{i\}|),
$$

where both $f^{T}$ and $f^{S}$ are increasing functions, and $\gamma$ is a real number in $(0,1)$. Pricing is similar to the case of the telephone, as the standardization effect appears on the utility functions of both buyers and non-buyers. The standardization effect makes the price level higher for each of the possible cases of the telephone.

\section{Results II: Endogenizing quality}

\subsection{General $\lambda$ functions}

Assume now the producer could choose a quality $\theta$ before he chooses a price $p(\theta)$. Afterwards, consumers decide to buy or not the product as in a demand configuration and beliefs by the producer about final demand are network consistent. With all these elements, we can say the following about the quality choice by the monopolist.

Proposition 4.1 Let $\lambda^{\max }(B)$ and $\lambda^{\min }(\neg B)$ for any set of buyers $B \in N$ be as defined before. Let $c_{0}$ be the marginal cost coefficient that makes the producer indifferent between providing a zero quality level and providing $\theta_{0}$, with corresponding optimal prices. Then $p^{*}=\bar{\lambda} \theta^{*}+[1-\bar{\lambda}] \theta_{0}$, for some $\bar{\lambda} \in[0,1]$, and:

1. If $c \geq \lambda^{\max }(N)$ then $\theta^{*}=0$.

2. If $\max _{B \subset N: \lambda^{\min }(B)>\lambda^{\max }(B)} \lambda^{\min }(B)<c \leq \lambda^{\max }(N)$ then:

$$
\theta^{*}=\left\{\begin{array}{cl}
0, & \text { if } c \geq c_{0} \\
\theta_{0}, & \text { if } c \leq c_{0}
\end{array}\right.
$$


3. Finally, if $0<c \leq \max _{B \subset N: \lambda^{\min }(B)>\lambda^{\max }(\neg B)} \lambda^{\min }(B)$ then there are threshold levels $\theta_{1}$ and $\theta_{2}$, both multiples of $\theta_{0}$ and greater than $\theta_{0}$, such that, given the same value $c_{0}$ as before:

$$
\theta^{*}=\left\{\begin{array}{cc}
0, & \text { if } c \geq c_{0} \text { and } \theta^{\max } \leq \theta_{1}, \\
\theta_{0}, & \text { if } c \leq c_{0} \text { and } \theta^{\max } \leq \theta_{2}, \\
\theta^{\max } & \text { either if } c \geq c_{0} \text { and } \theta^{\max } \geq \theta_{1}, \text { or if } c \leq c_{0} \text { and } \theta^{\max } \geq \theta_{2} .
\end{array}\right.
$$

Proposition 4.1 states the following. If the impact of quality on marginal costs $c$ is higher than the highest network effect, the producer has no incentives to provide a good with positive network externalities on utility. For intermediate values of $c$, where the limits depend on the network effects of the market, the producer is willing to provide at most the quality level corresponding to zero network network effect given by $\theta_{0}$. Finally, for low values of $c$, or, the other way around, for $\lambda$ functions high enough, the producer chooses $\theta^{\max }$ if $\theta^{\max }$ is above a certain threshold level. If $\theta^{\max }$ is below such a threshold level the the producer will choose quality levels equal to $\theta_{0}$ or 0 depending on which one is the most profitable one. Note that a $c$ bigger than $c_{0}$ means that a quality of 0 is more profitable than $\theta_{0}$, and viceversa. This parameter $c_{0}$ depends on the structure of the network, and, as shown in a working paper version of this paper, it might not be monotonic on the density of the network. ${ }^{7}$

\subsection{Particular cases}

For the sake of completeness, I provide the solution for the particular cases presented in the previous section. Since all those examples verify that $\theta_{0}=0$ we know that $\theta_{1}=\theta_{2}=0$.

- The telephone market

1. The star network: $\theta^{*}=\theta^{\max }$ if $c \leq f(1)$ and $\rho \geq 0$. Otherwise, $\theta^{*}=0$.

2. The regular network of degree $k: \theta^{*}=\theta^{\max }$ if $c \leq f(k)$ and $\rho \geq 0$. Otherwise, $\theta^{*}=0$.

3. The core-periphery network: $\theta^{*}=\theta^{\max }$ if $c \leq f(n-1)$ and $\rho_{n} \geq 0$ or if $c \leq f(\alpha-1)$ and $q_{\alpha}^{e} \geq 0$. Otherwise, $\theta^{*}=0$.

- Standardized market: $\theta^{*}=\theta^{\max }$ if $c \leq f(n-1)$ and $\rho \geq 0$. Otherwise, $\theta^{*}=0$.

\section{Conclusion}

I have presented a model to study pricing and quality decision under network effects by a monopolist. The main results characterize the choice of price and quality, respectively, by the

\footnotetext{
${ }^{7}$ One network has higher density than another if everybody has at least the same number of links in the former than in the latter, and at least one agent has strictly more links.
} 
producer in terms of threshold levels for the impact of quality on marginal costs. I obtain that higher prices are associated to higher quality only if network effects are high enough, provided the impact of quality on marginal costs lie in a middle range, or if the monopolist is optimistic enough about the size of final demand. As an implication, the incentives for the monopolist to provide higher valued goods by consumers are positive when the network effect is high enough or the monopolist is optimistic enough about the size of final demand. On the one hand, if the network effect is high enough consumers are ready to pay more as the size of the market increases and on the other hand, if the monopolist is optimistic about the size of final demand, he will expect higher profits from a given quality-price combination. Nevertheless, if marginal costs are low enough the monopolist might prefer charging the lowest price, therefore giving up the premium for quality, in order to capture all the market.

\section{References}

[1] Ambrus, A. \& R. Argenziano (2009), Asymmetric Networks in Two-Sided Markets. American Economic Journal: Microeconomics 1(1): 17-52.

[2] Argenziano, R. (2008), Differentiated Networks: Equilibrium and Efficiency. The RAND Journal of Economics 39(3): 747-769.

[3] Bagwell, K. \& M. H. Riordan (1991), High and Declining Prices Signal Product Quality. American Economic Review 81: 224-239.

[4] Becker, G. S. (1991), A Note on Restaurant Pricing and Other Examples of Social Influences on Price. Journal of Political Economy 99(5): 1109-1116.

[5] Bensaid, B. \& J.-P. Lesne (1996), Dynamic Monopoly Pricing with Network Extemalities. International Journal of Industrial Organization 14: 837-855.

[6] Besen, S.M. \& J. Farrell (1994), Choosing How to Compete: Strategies and Tactics in Standardization. Journal of Economic Perspectives 8: 117-131.

[7] Cabral, L.M.B. (2011), Dynamic Price Competition with Network Effects. Review of Economic Studies 78: 83-111.

[8] Cabral, L.M.B., D.J. Salant \& G.A. Woroch (1999), Monopoly Pricing with Network Externalities. International Journal of Industrial Organization 17: 199-214.

[9] Economides, N. (1996), The Economics of Networks. International Journal of Industrial Organization 14: 673-699.

[10] Farrell, J. \& G. Saloner (1985), Standardization, Compatibility, and Innovation. The RAND Journal of Economics 16: 70-83 
[11] Farrell, J. \& G. Saloner (1986), Installed Base and Compatibility: Innovation, Product Preannouncements, and Predation. American Economic Review 76: 940-955.

[12] Fudenberg, D. \& J. Tirole (2000), Pricing a Network Good to Deter Entry. The Journal of Industrial Economics 48: 373-390.

[13] Gul, F., H. Sonnenschein \& R. Wilson (1986), Foundations of Dynamic Monopoly and the Coase Conjecture. Journal of Economic Theory 39: 155-190.

[14] Jackson, M.O. (2008), Social and Economic Networks. Princeton University Press: Princeton.

[15] Jackson, M.O. \& A. Wolinsky (1996), A Strategic Model of Social and Economic Networks. Journal of Economic Theory 71: 44-74.

[16] Jing, B. (2007), Network Externalities and Market Segmentation in a Monopoly. Economic Letters 95: 7-13.

[17] Karni, E. \& D. Levin (1994), Social Attributes and Strategic Equilibrium: A Restaurant Pricing Game. Journal of Political Economy 102(4): 822-840.

[18] Katz, M.L. \& C. Shapiro (1985), Network Externalities, Competition, and Compatibility. American Economic Review 75: 424-440.

[19] Katz, M.L. \& C. Shapiro (1986), Technology Adoption in the Presence of Network Externalities. Journal of Political Economy: 822-841.

[20] Katz, M.L. \& C. Shapiro (1992), Product Introduction with Network Externalities. Journal of Industrial Economics 40: 55-83.

[21] Katz, M.L. \& C. Shapiro (1994), Systems Competition and Network Effects. Journal of Economic Perspectives 8: 93-115.

[22] Liebesking, J. \& R.P. Rumelt (1989), Markets for Experience Goods with Performance Uncertainty. The RAND Journal of Economics 20: 601-621.

[23] Liebowitz, S.J. \& S.E. Margolis (1994), Network Externality: An Uncommon Tragedy. Journal of Economic Perspectives 8(2): 133-150.

[24] Milgrom, P. \& J. Roberts (1986), Price and Advertising Signals of Product Quality. Journal of Political Economy 94: 796-821.

[25] Navarro, N. (2006), Asymmetric Information, Word-of-mouth and Social Networks: from the market for lemons to efficiency. CORE Discussion Paper 2006/02. Universit Catholique de Louvain. 
[26] Navarro, N. (2008), Quality Provision under Referral Consumption. Malaga Economic Theory Research Center Working Papers WP 2008-12.

[27] Oren, S.S. \& S.A. Smith (1981), Critical Mass and Tariff Structure in Electronic Communications Markets. The Bell Journal of Economics 12(2): 467-487.

[28] Pigeard de Almeida Prado, F., V. Belitsky \& Luiz Ferreira, A. (2011), Social Interactions, Product Differentiation and Discontinuity of Demand. The Journal of Mathematical Economics 47: 642-653.

[29] Rohlfs, J. (1974), A Theory of Interdependent Demand for a Communications Service. Bell Journal of Economics and Management Science 5: 16-37.

[30] Shapiro, C. (1982), Consumer Information, Product Quality, and Seller Reputation. The Bell Journal of Economics 13: 20-35.

[31] Shapiro, C. (1983a), Optimal Pricing of Experience Goods. The Bell Journal of Economics 14: 497-507.

[32] Shapiro, C. (1983b), Premiums for High Quality Products as Returns to Reputations. The Quarterly Journal of Economics 98: 659-679.

[33] Shy, O. (2011), A Short Survey of Network Economics. The Review of Industrial Organization 38: 119149.

[34] Vettas, N. (1997), On the Informational Role of Quantities: Durable Goods and Consumers' Word-of-Mouth Communication. International Economic Review 38(4): 915-944.

[35] Vettas, N. (1998), Demand and Supply in New Markets: Diffusion with Bilateral Learning. RAND Journal of Economics 29: 215-233.

[36] Wolinsky, A. (1983), Price as Signals of Product Quality. Review of Economic Studies 50: 647-658.

[37] Yang, J.-H.S. (2009), Social Network Influence and Market Instability. The Journal of Mathematical Economics 45: 257-276. 


\section{Appendix}

Proposition 3.1 characterizes the choice of price for a given quality, as a function of the parameters. Proposition 4.1 characterizes, respectively, the equilibrium with endogenous quality choice, also as a function of the parameters. As a previous step, Lemma 1 characterizes the structure of the demand configurations for any possible choice of quality and price by the producer. Lemma 1 not only helps proving the results stated in Proposition 3.1 and Proposition 4.1 but it also helps understanding the equilibrium behavior for consumers.

Lemma 1 Let $\theta$ and $p$ be the choices of quality and price made by the producer.

1. Assume that $\theta=\theta_{0}$.

(a) If $0 \leq p \leq \theta_{0}$ then $Q(p)=\{N\}$ and $q^{d}(p)=n$.

(b) If $p>\theta_{0}$ then $Q(p)=\{\emptyset\}$ and $q^{d}(p)=0$.

2. Assume that $\theta>\theta_{0}$.

(a) If $0 \leq p \leq \theta_{0}$ then $Q(p)=\{N\}$ and $q^{d}(p)=n$.

(b) If $p>\theta_{0}$ then, there exist numbers $\left\{\lambda_{k}\right\}_{k=1}^{K}$ with $0=\lambda_{1} \leq \lambda_{2} \leq \ldots \leq \lambda_{k} \leq \ldots \leq \lambda_{K} \leq$ 1 such that

i. if $\lambda_{k} \theta+\left(1-\lambda_{k}\right) \theta_{0}<p \leq \lambda_{k+1} \theta+\left(1-\lambda_{k+1}\right) \theta_{0}$ then $Q(p)$ keeps the same structure and therefore $q^{d}(p)=q_{k+1}^{H}$ is constant, for $k \leq K-1$.

ii. if $p>\lambda_{K} \theta+\left(1-\lambda_{K}\right) \theta_{0}$ then $Q(p)=\{\emptyset\}$ and $q^{d}(p)=0$.

3. Assume that $\theta<\theta_{0}$. Then, there exist numbers $\left\{\lambda_{k}\right\}_{k=1}^{K}$ with $0 \leq \lambda_{K} \leq \lambda_{K-1} \leq \ldots \leq \lambda_{1}=$ $\lambda^{\max }(N) \leq 1$

(a) If $0 \leq p \leq \lambda_{1} \theta+\left(1-\lambda_{1}\right) \theta_{0}$ then $Q(p)=\{N\}$ and $q^{d}(p)=q_{1}^{L}=n$.

(b) If $\lambda_{k} \theta+\left(1-\lambda_{k}\right) \theta_{0}<p \leq \lambda_{k+1} \theta+\left(1-\lambda_{k+1}\right) \theta_{0}$ then $Q(p)$ keeps the same structure and $q^{d}(p)=q_{k+1}^{L}$ is constant.

(c) If $p>\theta_{0}$ then $Q(p)=\{\emptyset\}$ and $q^{d}(p)=0$.

The proof of Lemma 1 is available at the web appendix.

\section{Proof of Proposition 3.1.}

We separate three cases as in Lemma 1. 
1. If $\theta=\theta_{0}$ the producer can at most expect a profit of $n(1-c) \theta_{0}>0$ by Lemma 1 , which is obtained by choosing a price equal to $\theta_{0}$

2. If $\theta>\theta_{0}$ let us define $\Lambda^{H}$ to be the set $\left\{\lambda_{k}\right\}_{k=1}^{K}$ with $0=\lambda_{1} \leq \lambda_{2} \leq \ldots \leq \lambda_{k} \leq \ldots \leq \lambda_{K} \leq 1$ such that (see Lemma 1)

(a) if $\lambda_{k} \theta+\left(1-\lambda_{k}\right) \theta_{0}<p \leq \lambda_{k+1} \theta+\left(1-\lambda_{k+1}\right) \theta_{0}$ then $Q(p)$ keeps the same structure. By network consistent beliefs we can define $q\left(\lambda_{k+1}\right)=q^{d}(p)$ when $\lambda_{k} \theta+\left(1-\lambda_{k}\right) \theta_{0}<$ $p \leq \lambda_{k+1} \theta+\left(1-\lambda_{k+1}\right) \theta_{0}$, for each $k \leq K-1$.

(b) if $p>\lambda_{K} \theta+\left(1-\lambda_{K}\right) \theta_{0}$ then $Q(p)=\{\emptyset\}$ and $q^{d}(p)=0$.

With all this, the highest maximum expected profit is given by

$$
\max \left\{n\left(\theta_{0}-c \theta\right), q^{*}\left[\left(\lambda^{*}-c\right) \theta+\left(1-\lambda^{*}\right) \theta_{0}\right], 0\right\}
$$

where $\lambda^{*}=\max _{\lambda \in \Lambda^{H}} q(\lambda)\left[(\lambda-c) \theta+(1-\lambda) \theta_{0}\right]$ and $q^{*}=q\left(\lambda^{*}\right)<n$.

Therefore,

(a) If $c \leq \frac{\theta_{0}}{\theta}-\frac{\lambda^{*} q^{*}}{n-q^{*}} \frac{\theta-\theta_{0}}{\theta}$ the producer's maximum expected profit is $n\left(\theta_{0}-c \theta\right)$, which is obtained by choosing a price equal to $\theta_{0}$.

(b) If $\frac{\theta_{0}}{\theta}-\frac{\lambda^{*} q^{*}}{n-q^{*}} \frac{\theta-\theta_{0}}{\theta} \leq c \leq \lambda^{*}+\left(1-\lambda^{*}\right) \frac{\theta_{0}}{\theta}$ the producer's maximum expected profit is $q^{*}\left[\left(\lambda^{*}-c\right) \theta+\left(1-\lambda^{*}\right) \theta_{0}\right]$, which is obtained by choosing a price equal to $\lambda^{*} \theta+(1-$ $\left.\lambda^{*}\right) \theta_{0}$, for $\lambda^{*} \in \Lambda^{H}$. Since $\theta>\theta_{0}$, this price is higher than $\theta_{0}$.

(c) Finally, if $c \geq \frac{\theta_{0}}{\theta}$ the producer's maximum expected profit is 0 , which is obtained by not producing at all.

By fixing $c_{1}=\frac{\theta_{0}}{\theta}-\frac{\lambda^{*} q^{*}}{n-q^{*}} \frac{\theta-\theta_{0}}{\theta}$ and $c_{2}=\lambda^{*}+\left(1-\lambda^{*}\right) \frac{\theta_{0}}{\theta}$ the corresponding statement of Proposition 3.1 follows.

3. If $\theta<\theta_{0}$ let us define $\Lambda^{L}$ to be the set $\left\{\lambda_{k}\right\}_{k=1}^{K+1}$ with $0=\lambda_{K+1} \leq \lambda_{K} \leq \lambda_{K-1} \leq \ldots \leq$ $\lambda_{1}=\lambda^{\max }(N)$ such that (see Lemma 1)

(a) If $0 \leq p \leq \lambda_{1} \theta+\left(1-\lambda_{1}\right) \theta_{0}$ then $Q(p)=\{N\}$ and $q^{d}(p)=q\left(\lambda_{1}\right)=n$.

(b) If $\lambda_{k} \theta+\left(1-\lambda_{k}\right) \theta_{0}<p \leq \lambda_{k+1} \theta+\left(1-\lambda_{k+1}\right) \theta_{0}$ then $Q(p)$ keeps the same structure. Again by network consistent beliefs we can define $q\left(\lambda_{k+1}\right)=q^{d}(p)$ for each $\lambda_{k} \theta+$ $\left(1-\lambda_{k}\right) \theta_{0}<p \leq \lambda_{k+1} \theta+\left(1-\lambda_{k+1}\right) \theta_{0}$.

(c) If $p>\theta_{0}$ then $Q(p)=\{\emptyset\}$ and $q^{d}(p)=0$. 
With all this, the highest expected profit is given by $q^{*}\left[\left(\lambda^{*}-c\right) \theta+\left(1-\lambda^{*}\right) \theta_{0}\right]$, for $\lambda^{*}=$ $\max _{\lambda \in \Lambda^{L}} q(\lambda)\left[(\lambda-c) \theta+(1-\lambda) \theta_{0}\right]$ and $q^{*}=q^{d}\left(\lambda^{*}\right)<n$. Note that $\left(\lambda^{*}-c\right) \theta+\left(1-\lambda^{*}\right) \theta_{0}>$ 0 given that $\theta_{0}>\theta$ and $c<1$. This means that the producer always produces in this case. Recall that $q(0) \geq 1$ is the expected demand when the price is equal to $\theta_{0}$.

Summarizing,

(a) If $c \geq \frac{\theta_{0}}{\theta}-\frac{q^{*} \lambda^{*}}{q^{*}-q(0)}$ the producer's maximum expected profit is $q(0)\left(\theta_{0}-c \theta\right)$, which is obtained by choosing a price equal to $\theta_{0}$.

(b) Otherwise, if $c \leq \frac{\theta_{0}}{\theta}-\frac{q^{*} \lambda^{*}}{q^{*}-q(0)}$ the producer's maximum expected profit is $q^{*}\left[\left(\lambda^{*}-c\right) \theta+\left(1-\lambda^{*}\right) \theta_{0}\right]$, which is obtained by choosing a price equal to $\lambda^{*} \theta+\left(1-\lambda^{*}\right) \theta_{0}$, for $\lambda^{*} \in \Lambda^{L}$. Since $\theta<\theta_{0}$, this price is smaller than $\theta_{0}$.

By fixing $c_{3}=\frac{\theta_{0}}{\theta}-\frac{q^{*} \lambda^{*}}{q^{*}-q(0)}$ the corresponding statement of Proposition 3.1 follows.

This proves Proposition 3.1

\section{Proof of Proposition 4.1.}

I provide here a shorter version of this proof. A more detailed version, including proofs for the claims that will follow, is available in the web appendix for the interested reader.

Since quality is endogenous from now on, $Q(p)$ will be denoted by $Q(\theta, p)$ and $q^{d}(p)$ will be denoted by $q^{d}(\theta, p)$. Let $\pi^{*}(\theta)$ denote the maximum value of profit for a given quality level $\theta$ and given consistent beliefs. In other words,

$$
\pi^{*}(\theta)=\max _{p} q^{d}(\theta, p)(p-c \theta) .
$$

Note first that I can write

$$
\pi^{*}(\theta)= \begin{cases}\pi_{H}^{*}(\theta), & \text { if } \theta>\theta_{0}, \\ \pi\left(\theta_{0}\right), & \text { if } \theta=\theta_{0}, \\ \pi_{L}^{*}(\theta), & \text { otherwise }\end{cases}
$$

where $\pi_{H}^{*}(\theta)=\max _{p} q^{d}(\theta, p)(p-c \theta), \pi_{L}^{*}(\theta)=\max _{p} q^{d}(\theta, p)(p-c \theta)$, and $\pi\left(\theta_{0}\right)=n(1-c) \theta_{0}$. The following two claims describe the shape and behavior of the $\pi^{*}$ function.

Claim 1. $\pi_{H}^{*}(\theta)$ tends to $\pi\left(\theta_{0}\right)$ as $\theta$ tends to $\theta_{0}$ and is continuous on $\left(\theta_{0}, \theta^{\max }\right]$. Furthermore, there are thresholds $c_{H}$ and $\theta^{H}$ such that

(i) if $c_{H}>c$ and $\theta^{\max }>\theta^{H}$ then the function $\pi_{H}^{*}(\theta)$ decreases to the right of $\theta_{0}$ until it reaches a minimum in between $\theta_{0}$ and $\theta^{\max }$ and increases all the way to the right until it reaches $\theta^{\max }$, and 
(ii) the function $\pi_{H}^{*}(\theta)$ is decreasing in $\left(\theta_{0}, \theta^{\max }\right]$ otherwise.

Claim 2. $\pi_{L}^{*}(\theta)$ tends to $\pi\left(\theta_{0}\right)$ as $\theta$ tends to $\theta_{0}$ and it is continuous in $\left[0, \theta_{0}\right)$. Furthermore,

(i) if $c<\lambda^{\max }(N)$ then $\pi_{L}^{*}(\theta)$ is increasing in a neighborhood to the left of $\theta_{0}$, with at most one local minimum in $\left(0, \theta_{0}\right)$, and

(ii) the function $\pi_{L}^{*}(\theta)$ is decreasing in $\left[0, \theta_{0}\right)$ otherwise.

From Claims 1 and 2 we can conclude that $\pi^{*}(\theta)$ is a continuous function, but we need to distinguish several cases. First note that from the proof of Claim 1 the threshold $c_{H}$ is in fact either a $\lambda^{\min }(B)$ or a $\lambda^{\max }(\neg B)$ for a $B \subset N$ that is a demand configuration. By monotonicity of the $\lambda$ functions, $\lambda^{\min }(B) \leq \lambda^{\min }(N) \leq \lambda^{\max }(N)$ and $\lambda^{\max }(\neg B)=\max _{i \notin B} \lambda_{i}(B, g) \leq$ $\max _{i \notin B} \lambda_{i}(N, g) \leq \lambda^{\max }(N)$. This means that if $c>\lambda^{\max }(N)$ then $c$ has to be greater than the threshold $c_{H}$. All this implies that if $c>\lambda^{\max }(N)$ both $\pi_{H}^{*}$ and $\pi_{L}^{*}$ are decreasing functions on their respective domains. We only then need to consider 2 cases.

1. If $c>\lambda^{\max }(N)$ then the function $\pi^{*}$ is decreasing in $\left[0, \theta^{\max }\right]$.

2. If $\lambda^{\max }(N) \geq c$ then the function $\pi^{*}$ has a local maximum at $\theta=\theta_{0}$.

Let $\theta^{*}$ be the global maximum of $\pi^{*}$, where $\pi^{*}$ is defined on the interval $\left[0, \theta^{\max }\right]$. Consider case 1 . Since the function $\pi^{*}$ is decreasing on its domain, we know that the only global maximum is $\theta^{*}=0$.

Consider now case 2 . We have a local maximum of $\pi^{*}$ at $\theta_{0}, \pi^{*}\left(\theta_{0}\right)=n(1-c) \theta_{0}$. In order to determine whether $\theta_{0}$ is a global maximum or not, we will have to compare this value with the values at the limits of the interval: $\pi^{*}(0)$ and $\pi^{*}\left(\theta^{\max }\right)$. The value of $c_{0}$ is found from $\pi^{*}(0)=\pi^{*}\left(\theta_{0}\right)$. Afterwards it is easy to check that $\pi^{*}(0) \geq \pi^{*}\left(\theta_{0}\right)$ if and only if $c \geq c_{0}$

For the case when the function $\pi_{H}^{*}$ is decreasing on its domain, we know that the global maximum will be either $\theta_{0}$ or 0 , but never $\theta^{\max }$. For the case when the function $\pi_{H}^{*}$ is increasing at some point in its domain, the values $\theta_{1}$ and $\theta_{2}$ are defined as the quality levels such that $\pi^{*}\left(\theta_{1}\right)=\pi^{*}(0)$ and $\pi^{*}\left(\theta_{2}\right)=\pi^{*}\left(\theta_{0}\right)$, both greater than $\theta_{0}$. If the function $\pi_{H}^{*}$ is increasing at some point $\hat{\theta}$ it will never be decreasing at any point $\theta>\hat{\theta}$, so for any $\theta^{\max } \geq \theta_{1}$ it is true that $\pi^{*}\left(\theta^{\max }\right) \geq \pi^{*}(0)$ and that for any $\theta^{\max } \geq \theta_{2}$ it is true that $\pi^{*}\left(\theta^{\max }\right) \geq \pi^{*}\left(\theta_{0}\right)$. Furthermore, $c \geq c_{0}$ if and only if $\pi^{*}(0) \geq \pi^{*}\left(\theta_{0}\right)$, which in turn implies that $\theta_{1} \geq \theta_{2}$. Summarizing for the case when $\lambda^{\max }(N) \geq c$ we obtain the following.

- if $c \geq c_{0}$, then $\pi^{*}(0) \geq \pi^{*}\left(\theta_{0}\right)$ and $\theta_{1} \geq \theta_{2}$

- if $\theta^{\max } \geq \theta_{1}$ then $\pi^{*}\left(\theta^{\max }\right) \geq \pi^{*}(0)$ 
- if $\theta^{\max } \geq \theta_{2}$ then $\pi^{*}\left(\theta^{\max }\right) \geq \pi^{*}\left(\theta_{0}\right)$

$\theta^{\max }$ is the global maximum when $\pi^{*}\left(\theta^{\max }\right) \geq \max \left\{\pi^{*}(0), \pi^{*}\left(\theta_{0}\right)\right\}, \theta_{0}$ is the global maximum when $\pi^{*}\left(\theta_{0}\right) \geq \max \left\{\pi^{*}(0), \pi^{*}\left(\theta^{\max }\right)\right\}$, and finally, 0 is the global maximum when $\pi^{*}(0) \geq$ $\max \left\{\pi^{*}\left(\theta^{\max }\right), \pi^{*}\left(\theta_{0}\right)\right\}$. Technical details can be checked, as said before, in the web appendix, where a more detailed version of this proof is available. 